\title{
Multimodality Imaging of Prostate Cancer
}

\author{
Soleen Ghafoor ${ }^{1}$, Irene A. Burger ${ }^{2}$, and Alberto H. Vargas ${ }^{1}$ \\ ${ }^{1}$ Department of Radiology, Memorial Sloan Kettering Cancer Center, New York, New York; and ${ }^{2}$ Department of Nuclear Medicine, \\ Baden Cantonal Hospital, Baden, Switzerland
}

\begin{abstract}
Learning Objectives: On successful completion of this activity, participants should be able to describe (1) the various distinct clinical scenarios for prostate cancer; (2) the various imaging modalities available for use in prostate cancer; (3) the role of these imaging modalities in primary or recurrent prostate cancer and their advantages and disadvantages; and (4) the future potential of theranostic approaches and the evolving indications for contemporary hybrid imaging modalities such as PET/MRI and whole-body MRI.
\end{abstract}

Financial Disclosure: Dr. Burger has received research grants and speaker honorarium from GE Healthcare. The authors of this article have indicated no other relevant relationships that could be perceived as a real or apparent conflict of interest.

CME Credit: SNMMI is accredited by the Accreditation Council for Continuing Medical Education (ACCME) to sponsor continuing education for physicians. SNMMI designates each JNM continuing education article for a maximum of 2.0 AMA PRA Category 1 Credits. Physicians should claim only credit commensurate with the extent of their participation in the activity. For CE credit, SAM, and other credit types, participants can access this activity through the SNMMI website (http://www.snmmilearningcenter.org) through October 2022.

Prostate cancer is a very heterogeneous disease, and contemporary management is focused on identification and treatment of the prognostically adverse high-risk tumors while minimizing overtreatment of indolent, low-risk tumors. In recent years, imaging has gained increasing importance in the detection, staging, posttreatment assessment, and detection of recurrence of prostate cancer. Several imaging modalities including conventional and functional methods are used in different clinical scenarios with their very own advantages and limitations. This continuing medical education article provides an overview of available imaging modalities currently in use for prostate cancer followed by a more specific section on the value of these different imaging modalities in distinct clinical scenarios, ranging from initial diagnosis to advanced, metastatic castration-resistant prostate cancer. In addition to established imaging indications, we will highlight some potential future applications of contemporary imaging modalities in prostate cancer.

Key Words: PET/MRI; fluciclovine; multiparametric MRI; PSMA; theranostics; whole-body MRI

J Nucl Med 2019; 60:1350-1358

DOI: 10.2967/jnumed.119.228320

\section{$\mathbf{P}$} rostate cancer $(\mathrm{PCa})$ is the most common noncutaneous malignancy in men in the Western world. $\mathrm{PCa}$ is characterized by a classic model of tumor heterogeneity, with a variety of clinical phenotypic states underpinned by histopathologic and molecular heterogeneity (1). Clinical presentations in patients-who are considered to bear the same disease under traditional definitionsrange from indolent, clinically insignificant cancers managed expectantly (active surveillance) to very aggressive, rapidly spreading forms with fatal outcomes.

The highly heterogeneous nature of PCa accounts for the ongoing complexity and challenges in its clinical management. Intense

Received Jun. 27, 2019; revision accepted Aug. 29, 2019.

For correspondence or reprints contact: Soleen Ghafoor, Memorial Sloan Kettering Cancer Center, 1275 York Ave., Box 29, New York, NY 10065.

E-mail: ghafoors@mskcc.org

Published online Sep. 3, 2019.

COPYRIGHT (C 2019 by the Society of Nuclear Medicine and Molecular Imaging. efforts have been undertaken to develop risk stratification tools to aid in clinical decision making and the optimization of patient care, usually by combining serum prostate-specific antigen (PSA) levels, histologic Gleason grade, and evaluation of the anatomic extent of the primary tumor.

Imaging plays a pivotal role in the management of $\mathrm{PCa}$ through its noninvasive approach to evaluating the presence and extent of local and distant disease. The most common sites of metastases are the lymph nodes (LNs) and bones. Visceral metastases are less commonly found and are usually associated with advanced, castration-resistant disease and with emergent histologic variants after multiple lines of prior treatment (2).

Multiple imaging modalities are used in different clinical scenarios, with marked geographic variations in use related to loosely defined indications, availability, costs, and regulatory restrictions. The ongoing refinements of imaging technology have changed the landscape of PCa imaging, benefiting clinical decision making by allowing earlier detection of disease spread than was previously possible.

In the following sections, we will provide a general description of the most important imaging modalities used in $\mathrm{PCa}$. The role of these techniques in each PCa clinical state will be discussed, as supported by current scientific evidence.

\section{OVERVIEW OF AVAILABLE IMAGING MODALITIES}

\section{MRI}

Multiparametric MRI (mpMRI) of the Pelvis. MRI allows detailed anatomic assessment of the prostate with clear depiction of zonal anatomy and exquisite soft tissue resolution superior to any other imaging modality to date (Supplemental Fig. 1; supplemental materials are available at http://jnm.snmjournals.org). Prostate MRI is typically performed on a 1.5- or 3-T magnet using a multichannel pelvic phased-array receiver coil with or without an endorectal coil. The use of an endorectal coil increases signal-to-noise ratio and was historically considered advantageous, especially in selected cases (larger patients, older 1.5-T magnets), but it is also associated with patient discomfort, image artifacts, and longer examination times. There is currently no consensus on the use of an endorectal coil (3).

MpMRI of the prostate is based on the combination of anatomic (T1-weighted and multiplanar T2-weighted images) and functional sequences (diffusion-weighted imaging with apparent diffusion 
coefficient maps and dynamic contrast-enhanced imaging). The addition of these functional sequences increases the sensitivity and positive predictive value of MRI (4).

Optimal prostate mpMRI is largely dependent on the acquisition of high-quality images and the use of adequate technical equipment paired with the expertise of the interpreting radiologist. Now in its recently published updated version (version 2.1), the Prostate Imaging Reporting and Data System (PI-RADS) was originally developed in 2012 to overcome excessive variations in technical equipment, imaging acquisitions, image interpretation, and reporting between centers (5). Of note, PI-RADS is not aimed at increasing the overall detection rate of all types of PCa but is rather intended to detect clinically significant $\mathrm{PCa}$, which is defined as those tumors likely to affect the patient's life-span. Although there is no uniform definition for clinically significant PCa, the most commonly used definition is a tumor with a Gleason grade of at least 7 or a volume of at least $0.5 \mathrm{~cm}^{3}$.

Whole-Body (WB) MRI. WB MRI is an attractive technique because of its high soft-tissue contrast and consequently exquisite anatomic detail, and lack of ionizing radiation. WB MRI is excellent for imaging of bone marrow, nodal, soft-tissue, and visceral metastases as well as for local tumor staging if combined with the required pelvic sequences for the prostate (Supplemental Fig. 2). The combined use of anatomic sequences (T1-weighted and fatsuppressed T2-weighted imaging) and diffusion-weighted imaging transforms WB MRI into a hybrid technique, providing combined morphologic and functional information. Robust validation data are lacking, partly because of a lack of standardization in image protocols, acquisition, and interpretation (6). Therefore, efforts have been made to overcome these shortcomings by an international panel of expert oncologic imagers and oncologists who published the METastasis Reporting and Data System for Prostate Cancer (7). This white paper comprises consensus recommendations on performance, quality standards, and reporting of WB MRI to accommodate the increased use of WB MRI. Of note, the current literature suggests that WB MRI may be less sensitive to current PET technologies for detection of PCa $(8,9)$; however, more robust data are needed, particularly to evaluate a possible complementary role for MR and PET.

\section{CT}

CT is not considered the primary imaging method for $\mathrm{PCa}$, because of inferior soft-tissue contrast and lack of molecular information. The main role for CT is the evaluation of nodal and distant metastases, for which it has a low yield compared with more advanced hybrid imaging methods, such as PET/CT. A major disadvantage of CT (and MRI) for LN assessment is the reliance on morphologic criteria (e.g., shape and size) (10). LNs harboring metastatic disease may be of normal size, and enlargement of nonmetastatic LNs may be due to reactive hyperplasia in the setting of infection or inflammation. Nevertheless, because of its wide availability, limitations in regulatory approvals and funding for advanced imaging modalities, and relatively low cost, CT is still currently recommended in patients with intermediate- to high-risk $\mathrm{PCa}$ in the guidelines of the American Urological Association.

\section{Bone Scintigraphy (BS)}

BS using ${ }^{99 m} \mathrm{mc}-$ labeled phosphonates provides an overview of the entire skeleton by allowing visualization of the tracer incorporation into the hydroxyapatite matrix of bone in proportion to blood flow and bone remodeling activity, and inflammatory changes can therefore mimic metastases on BS (11).

\section{$\mathrm{PET} / \mathrm{CT}$}

$\mathrm{PET} / \mathrm{CT}$ has gained increasing importance in the imaging workup of PCa. Currently, there are several radiolabeled tracers in use that have demonstrated efficacy for cancer detection in various clinical settings. A detailed discussion of the different PET tracers would be well beyond the scope of this review, and commonly used PET tracers in imaging of PCa have been described in more detail in dedicated structured reviews (12).

In this first general section, selected PET tracers will be discussed to provide a short overview followed by an evidence-based, more detailed discussion on their specific use, advantages, and shortcomings with regard to the different clinical scenarios of PCa.

${ }^{18} \mathrm{~F}$-FDG PET/CT. Despite its widespread use in oncologic imaging, ${ }^{18} \mathrm{~F}-\mathrm{FDG}$ PET/CT does not play as important a role in the imaging of PCa because of its limited sensitivity in localized and early metastatic disease. Nonetheless, ${ }^{18}$ F-FDG PET/CT may be useful in specific scenarios along the natural history of PCa (13). Increased ${ }^{18} \mathrm{~F}$-FDG uptake seems to be more frequent in aggressive forms, aberrant histology (e.g., neuroendocrine), and advanced cases of metastatic castration-resistant PCa (mCRPC) $(14,15)$.

${ }^{18} \mathrm{~F}$-Sodium Fluoride (NaF) PET/CT. As with BS, ${ }^{18} \mathrm{~F}-\mathrm{NaF}$ uptake does not provide direct visualization of the presence of tumor cells but rather reflects the increased blood flow, bone remodeling, and osteoblastic activity related to osseous metastases. Compared with $\mathrm{BS},{ }^{18} \mathrm{~F}-\mathrm{NaF}$ has greater bone uptake and faster soft-tissue clearance (due to minimal binding to serum proteins), which translate to a higher bone-to-background contrast and shorter examination time (16). In a prospective study by Löfgren et al. (17), ${ }^{18} \mathrm{~F}-\mathrm{NaF}$ PET/CT resulted in reduced equivocal readings compared with BS. There is currently no evidence to support the clinical benefit of routine use of ${ }^{18} \mathrm{~F}-\mathrm{NaF}$ PET/CT over BS. Another limitation of ${ }^{18} \mathrm{~F}-\mathrm{NaF}$ PET/CT is its bone-only detection capability, which renders it a less appealing choice in the era of evolving targeted tracers for molecular imaging of PCa that can concurrently depict extraskeletal disease.

Choline PET/CT. Imaging with either ${ }^{11} \mathrm{C}$ - or ${ }^{18} \mathrm{~F}$-labeled choline is based on an increased uptake and turnover of phosphatidylcholine in cancer cells, which is an essential part of the phospholipids in the cellular membrane (18). The advantage of ${ }^{11} \mathrm{C}$ over ${ }^{18} \mathrm{~F}$ is a lower urinary excretion that facilitates evaluation of the prostate bed and lower patient exposure, but it is limited by a short half-life of 20 minutes, which necessitates an on-site cyclotron (19). The role for choline PET/CT is limited in diagnosis and primary staging of $\mathrm{PCa}$ because of its relatively low sensitivity; the main use of choline PET/CT lies in restaging in the setting of biochemical recurrence (BCR). It has been replaced by prostate-specific membrane antigen (PSMA) PET/CT in many institutions, albeit not in the United States and some other countries where regulatory approval for use of PSMA is pending. In the United States, ${ }^{11} \mathrm{C}$-choline PET/CT is currently performed by several institutions under an investigational new drug exemption issued by the U.S. Food and Drug Administration. A potential role for choline PET/CT may be in the small proportion of PSMAnegative PCa cases, although this indication has not yet been studied and requires extended evaluation.

${ }^{18} \mathrm{~F}$-Fluciclovine PET/CT. ${ }^{18} \mathrm{~F}$-fluciclovine (anti-1-amino-3- ${ }^{18} \mathrm{~F}-$ flurocyclobutane-1-carboxylic acid [Axumin; Blue Earth Diagnostics, Inc.]) is a non-naturally occurring L-leucine amino acid analog radiotracer. Imaging with ${ }^{18} \mathrm{~F}$-fluciclovine mirrors the upregulated transmembrane amino acid transport that occurs in PCa secondary to increased tumor-related metabolism and protein synthesis. 
Axumin was approved in 2016 by the Food and Drug Administration for PET imaging in men with suspected recurrence in the setting of BCR after prior treatment (Fig. 1; Supplemental Fig. 3), the groundwork for which was laid by the prospective study of Schuster et al. (20) showing high accuracy for this radiotracer in detection of recurrent PCa. It is currently not Food and Drug Administration-approved for primary staging or response assessment.

PSMA PET/CT. PSMA is a type II transmembrane glycoprotein with enzymatic carboxypeptidase activity that is expressed in the cytosol of normal prostatic tissue and highly overexpressed on the membrane in PCa cells (21). The extent of membranous PSMA expression correlates positively with tumor grade and rises under androgen deprivation and in metastatic and castration-resistant cancer, thus rendering it an ideal target for imaging and treatment (21).

PSMA seems to be an ideal target for several reasons: preferential, marked overexpression by most PCa cells, positive correlation of its expression with tumor grade and disease stage, low presence in the bloodstream by virtue of its transmembrane localization, and internalization and retention within tumor cells after binding to its ligand (22). Furthermore, in the era of personalized medicine, there is increased interest in the use of PSMA for therapeutic approaches, that is, in the theranostics setting, combining imaging diagnosis with targeted radionuclide therapy.

Although the name implies specificity, PSMA expression is by no means exclusive to the prostate. There is physiologic high expression in the kidneys and small intestines and abnormal expression in a variety of benign and malignant nonprostatic processes, such as benign skeletal abnormalities (fractures, fibrous dysplasia) and other cancers $(23,24)$. PSMA uptake in nonprostatic malignancies is related to PSMA expression of the tumor-related neovasculature (25). Nevertheless, because of

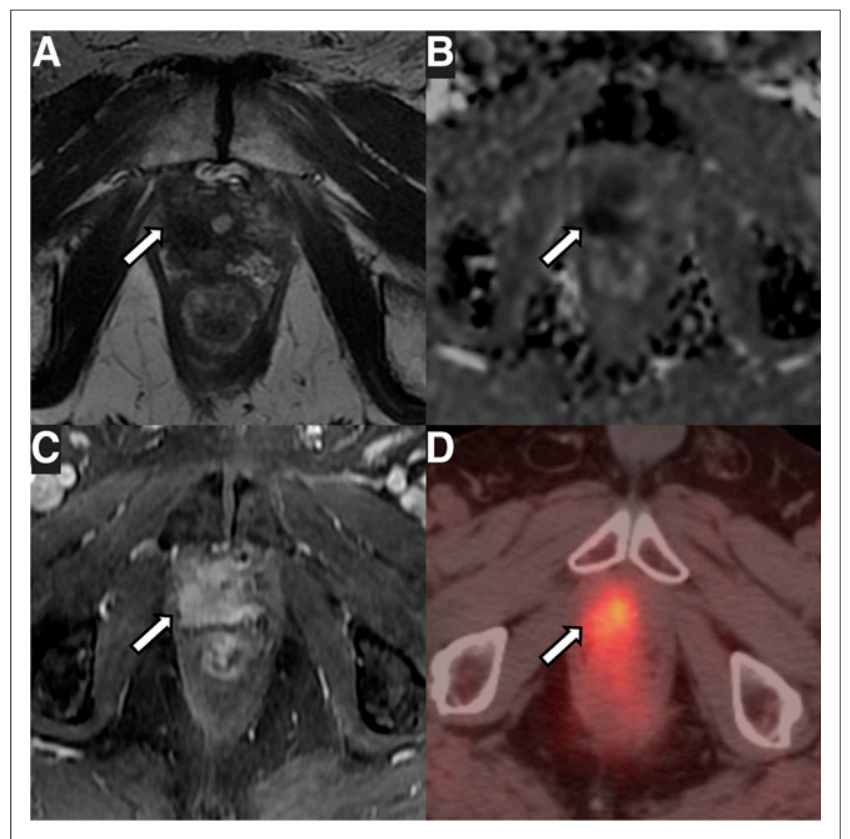

FIGURE 1. A 75-y-old patient with local recurrence of Gleason $3+4$ $\mathrm{PCa}$ (arrows) treated with radical prostatectomy. (A-C) mpMRI shows masslike T2-hypointense thickening at right lateral vesicourethral anastomosis (A) with diffusion restriction on apparent diffusion coefficient image (B) and early arterial enhancement on dynamic contrast-enhanced image (C). (D) ${ }^{18} \mathrm{~F}$-fluciclovine PET/CT shows asymmetric increased radiotracer uptake at that site $\left(S U V_{\max }, 5.5\right)$. its high sensitivity, PSMA has quickly become the front-runner of the currently available tracers for imaging of $\mathrm{PCa}$ and has shown a robust performance even at low PSA levels.

Currently, the most widely used PSMA tracer is ${ }^{68}$ Ga-PSMA-11; other commonly used tracers are radiofluorinated ${ }^{18} \mathrm{~F}$-DCFPyL-PSMA, ${ }^{18}$ F-DFCBC-PSMA, and ${ }^{18}$ F-PSMA-1007-the last of these being potentially interesting for future applications because of its predominantly hepatobiliary clearance, as opposed to the renal clearance of ${ }^{68} \mathrm{Ga}-\mathrm{PSMA}-11$, by which it could overcome some limitations in evaluation of local recurrence and locoregional pelvic nodal disease (26).

${ }^{18} \mathrm{~F}$-FDHT PET/CT. ${ }^{18} \mathrm{~F}-16 \beta$-fluoro-5 $\alpha$-dihydrotestosterone $\left({ }^{18} \mathrm{~F}-\mathrm{FDHT}\right)$ is of particular interest in the setting of advanced castration-resistant PCa. It targets the androgen receptor (AR), which with its native ligands testosterone and $5 \alpha$-dihydrotestosterone plays a pivotal role in the development of male sexual differentiation. Functional alterations of the $\mathrm{AR}$ are linked to the development and progression of PCa, rendering it an androgen-driven disease. Therefore, virtually all patients initially respond to androgen-deprivation therapy (ADT). However, nearly all patients will invariably progress to a castration-resistant state within 2-3 years despite low testosterone levels (27). This is due to incomplete depletion of androgens and persistent AR signaling through mechanisms such as mutations, overexpression, and ligand-independent $\mathrm{AR}$ activation promoting cancer growth and metastasis in those patients on conventional ADT (28).

A potential role for ${ }^{18} \mathrm{~F}$-FDHT PET/CT may be in advanced PCa refractory to initial conventional ADT. ${ }^{18} \mathrm{~F}-\mathrm{FDHT}$ PET/CT is currently exclusively used for investigational research purposes and has not been approved for clinical routine. Preliminary studies on the use of ${ }^{18} \mathrm{~F}$-FDHT PET/CT in patients with castrationresistant PCa demonstrated safety, feasibility, good accuracy of lesion detection, and association with survival $(29,30)$.

\section{PET/MRI}

Main advantages of PET/MRI over PET/CT are higher softtissue contrast and lower radiation dose to the patient. Challenges that once precluded the adoption of hybrid PET/MRI systems in clinical practice have recently been addressed whereas some remain, notably issues related to MR-attenuation correction and validation of quantitative uptake metrics (e.g., accuracy of SUV measurements). Operational challenges include the need for technologists appropriately trained in dual PET and MRI applications as well as training for hybrid imagers enabling interpretation of combined PET/MRI studies. The long-term challenges of PET/MRI use entail clinical validation through evidence of improved patient outcomes and incremental value when compared with PET/CT or mpMRI alone.

Several studies have shown the potential for improved lesion detection using combined PSMA PET/MRI for primary PCa (31-33). The combined PET/MRI approach has also been shown to increase the specificity of ${ }^{18} \mathrm{~F}$-fluorocholine PET, which is generally limited in specificity because of significant overlap in uptake between malignant and benign conditions (e.g., benign prostatic hyperplasia) (33).

\section{OVERVIEW OF POTENTIAL IMAGING INDICATIONS FOR DIFFERENT CLINICAL SCENARIOS}

\section{Imaging of the Primary Prostate Tumor}

Because of its superb soft-tissue resolution, MRI has established itself as the modality of choice for the assessment of primary prostate tumors and has been applied in clinical practice for over 3 decades. During most of this time, it was predominantly used in tertiary academic centers with special interest and expertise, but 
most recently its adoption has been more widespread, partly because of the introduction of PI-RADS in 2012 and the updated version 2 in 2015 and version 2.1 in 2019 (3,5). PI-RADS standardized image acquisition and reporting, with assessments based on the appearance of lesions on T2-weighted imaging, diffusion-weighted/ apparent diffusion coefficient imaging, and, as an ancillary adjunct, dynamic contrast-enhanced imaging. Prostate tumors typically demonstrate homogeneously low T2 signal intensity, diffusion restriction, and early enhancement (Fig. 2). Per-lesion assessment categories are assigned, ranging from 1-5 and reflecting the likelihood of clinically significant PCa. Thus, PI-RADS 1 suggests a very low probability of clinically significant cancer whereas PIRADS 5 suggests a very high probability. PI-RADS 3 defines an intermediate (and in clinical practice often translating into indeterminate) probability.

Two landmark papers providing level 1 evidence for the incremental benefit of mpMRI have substantiated the strength of MRI in the detection of clinically significant PCa, that is, the PROMIS and PRECISION trials $(34,35)$.

PROMIS (34) was a multicenter prospective trial comparing the accuracy of mpMRI versus transrectal ultrasound (TRUS)-guided biopsy to the reference standard of transperineal mapping biopsy in 576 biopsy-naïve patients with an elevated risk for PCa. MRI was found to be superior to standard TRUS-guided biopsy for the detection of clinically significant $\mathrm{PCa}$, missing only 17 of $230(7 \%)$ clinically significant cancers compared with 119 of 230 (52\%) clinically significant cancers by TRUS-guided biopsy. mpMRI was more sensitive than TRUS-guided biopsy (93\% for mpMRI vs. $48 \%$ for TRUS, $P<0.0001$ ) but less specific ( $41 \%$ for mpMRI vs.

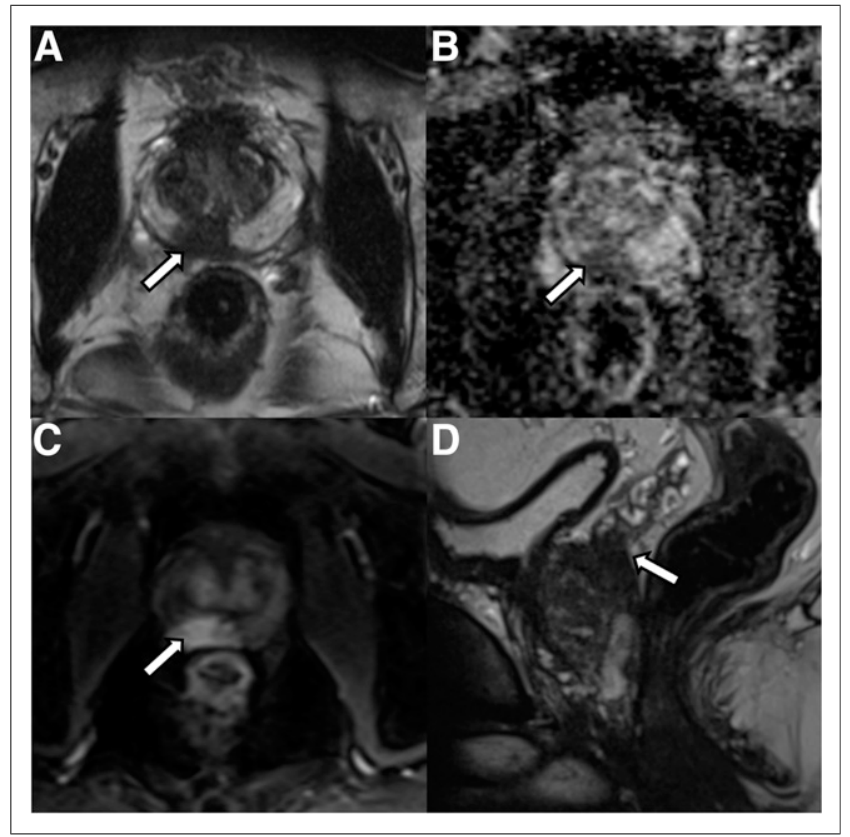

FIGURE 2. A 53-y-old patient with abnormal digital rectal findings, elevated PSA level, and lesion (arrows) found on imaging. (A-C) mpMRI shows circumscribed T2-hypointense lesion in right posterior base to mid gland peripheral zone (A) with marked diffusion restriction $(B)$ and early arterial enhancement (C). Lesion measured $1.9 \mathrm{~cm}$ and involved the central zone and base of seminal vesicles. Final surgical pathology revealed $3+4$ Gleason $\mathrm{PCa}$ with extracapsular extension and seminal vesicle invasion.
95\% for TRUS, $P<0.0001$ ), with a positive predictive value of $51 \%$ (95\% confidence interval, $46 \%-56 \%$ ) but a high negative predictive value for mpMRI of $89 \%$ (95\% confidence interval, $83 \%-94 \%$ ). Thus, given the high negative predictive value, biopsy could be avoided in $27 \%$ of patients if mpMRI were incorporated in the diagnostic work-up.

The PRECISION study (35) was a prospective multicenter randomized trial comparing MRI-targeted prostate biopsy with standard TRUS-guided biopsy for the detection of clinically significant cancer in at-risk biopsy-naïve men. In the MRI-targeted biopsy group, 38\% of clinically significant cancers were found, compared with $26 \%$ in the standard biopsy group, whereas fewer indolent cancers were detected in the mpMRI-targeted biopsy group than in the standard-biopsy group (9\% vs. $22 \% ; P<0.001)$. A potential limitation was that men with normal MRI results $(28 \%)$ did not undergo subsequent biopsy (as compared with the PROMIS trial, in which all patients underwent the transperineal mapping as a reference standard), thus leaving some uncertainty as to the true prevalence of disease in these patients without tissue sampling.

Overall, both studies conformed to the goals of contemporary PCa management—namely, to accurately find the "bad actors" while minimizing the detection of low-grade, low-volume indolent disease that is unlikely to result in adverse cancer-related events but may lead to overtreatment.

The value of mpMRI for ruling out clinically significant PCa was also evaluated in a large metaanalysis of 48 studies including 9,613 patients (36). The median negative predictive value of mpMRI for detecting clinically significant $\mathrm{PCa}$ was $88.1 \%$ (interquartile range, 85.7-92.3), although results varied widely because of heterogeneity in study design, inclusion criteria, preimaging risk stratification, definition of clinically significant cancer, and mpMRI reporting of positive findings.

Despite the high negative predictive value of mpMRI, clinically significant tumors can still be missed and the false-negative rate of mpMRI could be reduced further (37). False-negative mpMRI findings are commonly associated with smaller tumor size, multifocality, presence of postbiopsy or postinflammatory changes distorting normal zonal anatomy, and tumor location in the transition zone or anterior fibromuscular stroma (38). Herein lies the potential incremental value of PET/MRI: improving the detection rate for cancers that are commonly missed on mpMRI and at the same time offering a one-stop shop for staging of PCa (Supplemental Figs. 4 and 5). In a retrospective study by Hicks et al. (33) in which 32 patients with biopsy-proven PCa scheduled for radical prostatectomy were included, the authors looked at region-specific sensitivities and specificities of PSMA-11 PET/MRI compared with mpMRI. PSMA PET/MRI had higher sensitivity than mpMRI alone ( $74 \%$ vs. $50 \% ; P<0.001$ ), whereas both were equally specific.

\section{Imaging Evaluation of Extraprostatic Extension of Primary Tumor}

An important contribution of mpMRI in the setting of local staging is evaluation for the presence of extraprostatic extension, which can best be assessed on T2-weighted imaging as broad contact of the tumor with the prostatic capsule, bulging of the capsule beyond the expected boundary of the gland, obliteration of the rectoprostatic angle, and asymmetry of the neurovascular bundles. Signs of seminal vesicle invasion are low signal on T2weighted imaging in the seminal vesicle, tumor location at the prostate base, loss of normal tubular architecture of the seminal 
vesicle, and associated diffusion restriction (39). A systematic analysis by de Rooij et al. (40) showed moderate sensitivity but very high specificity and negative predictive value for mpMRI in prediction of extraprostatic extension and seminal vesicle invasion.

Although MRI has been the primary imaging modality used for the evaluation of extraprostatic extension, more recently there has been an interest in using combined PSMA PET/MRI for improved accuracy of local $\mathrm{T}$ staging and evaluation of extraprostatic extension (41).

\section{Imaging of Nodal Metastases at Initial Staging}

The probability for LN metastases at initial diagnosis of localized $\mathrm{PCa}$ is associated with risk classification; very low risk and low-risk patients (e.g., Gleason grade $\leq 6$, PSA $<10 \mathrm{ng} / \mathrm{mL}, \mathrm{T} 1-\mathrm{T} 2 \mathrm{a}$ stage) have an exceedingly low probability for LN metastasis (42). Accurate assessment of $\mathrm{LN}$ status helps in therapeutic decision making, prediction of recurrence risk, and assessment of prognosis.

The presence of LN metastasis is an independent risk factor for BCR (43), and there is great interest in preoperative imagingbased identification of LN metastases to optimize surgical and adjuvant or neoadjuvant treatment planning. Currently, the clinical practice and urologic guidelines primarily rely on validated clinical nomograms to help guide decision making for pelvic lymph node dissection, with a calculated estimated risk of more than $5 \%$ being an indication to perform extended pelvic lymph node dissection (44). Even though pelvic lymph node dissection is the traditional gold standard for LN staging, it is an invasive, time-consuming approach largely dependent on surgical skill and templates.

The role of imaging for the detection of $\mathrm{LN}$ metastases is controversial and still evolving. It is acknowledged that the low sensitivity of conventional modalities routinely leads to underestimation of the disease burden. Assessment criteria for suggestion of abnormal LNs on conventional imaging are based on morphology (e.g., size and shape), but almost $80 \%$ of LN metastases occur in small, subcentimeter LNs and there are also benign causes for enlarged LNs, rendering size-based criteria unreliable (45). A metaanalysis of 24 studies (46) found pooled sensitivities and specificities of $42 \%$ and $82 \%$, respectively, for CT and $39 \%$ and $82 \%$, respectively, for MRI for the detection of malignant LNs. A more recent metaanalysis of 24 studies with 2,928 patients on the performance of mpMRI for detection of LN metastases (47) came to a similar conclusion, finding only moderate sensitivity of $56 \%$ but high specificity of $94 \%$.

As of yet, no single imaging modality has shown optimal diagnostic performance in the assessment of metastatic LNs. Studies with the PET tracers ${ }^{18} \mathrm{~F}$-choline, ${ }^{11} \mathrm{C}$-choline, and ${ }^{18} \mathrm{~F}$-fluciclovine reported similar high specificities but low sensitivities ranging from $40 \%$ to $50 \%(48,49)$.

Several studies have established the value of PSMA PET in the evaluation of early BCR, and there are also increasing reports on the utility of PSMA PET for preoperative LN staging. An ongoing Australian phase III prospective randomized multicenter trial, the proPSMA study (Australian New Zealand Clinical Trials Registry 12617000005358), is under way and may provide better insight into the value of PSMA PET/CT in the primary staging setting (50). Another multicenter trial, in Germany, is prospectively investigating the accuracy of ${ }^{68} \mathrm{Ga}-\mathrm{PSMA}-11 \mathrm{PET} / \mathrm{CT}$ for primary staging of high-risk PCa patients (NCT03362359).

In a prospective study including 122 patients, Grubmüller et al. (51) evaluated the performance of ${ }^{68} \mathrm{Ga}$-PSMA-11 PET/MRI for preoperative staging of $\mathrm{PCa}$. They found a moderately high sensitivity, good specificity, and high accuracy for detection of LN metastases $(68.8 \%, 100 \%$, and $93 \%$, respectively). Those cases that were missed in imaging were all sized less than $4 \mathrm{~mm}$, indicating limited sensitivity for very small micrometastases. These findings are in line with the findings of Maurer et al. (52) (with sensitivity of $65.9 \%$ on patient-based analysis) and Giesel et al. (53), with the latter specifically emphasizing the superior performance of PSMA PET/CT in detecting smaller LNs that do not meet conventional morphologic criteria.

\section{Imaging of Distant Metastases at Initial Presentation}

The most common site of distant metastases in PCa is the skeletal system, but the prevalence is variable and dependent on risk categories. For example, in patients with newly diagnosed $\mathrm{PCa}$ undergoing preoperative staging mpMRI, the prevalence of bone metastases is only $1.5 \%$ (14). Therefore, whole body imaging (with any technique) is indicated only in patients with intermediate- or high-risk disease. Visceral metastases are rare at initial diagnosis.

Although current guidelines recommend conventional imaging modalities such as BS and CT for the detection of metastases, it is well recognized that these suffer from moderate sensitivity, a significant rate of equivocal findings, poor performance for PSA levels of less than $20 \mathrm{ng} / \mathrm{mL}$, and a lack of specificity. Therefore, more advanced techniques are being increasingly used (Supplemental Fig. 5). However, BS is established for treatment response assessment and has been shown to be an independent prognostic imaging biomarker of survival in patients with metastatic disease $(54,55)$.

An important role for imaging in advanced PCa is the identification of patients with unfavorable features, namely bone metastases beyond the axial skeleton, lytic-appearing bone metastases, visceral metastases, or bulky tumor masses (10). These patients with unfavorable features will likely benefit from intensified treatment regimens including targeted radiation therapy and chemotherapy (56).

A retrospective study of 126 patients by Pyka et al. (57) compared the diagnostic performance of ${ }^{68} \mathrm{Ga}$-PSMA PET with that of ${ }^{99 m}$ Tc-methyldiphosphonate BS for the detection of bone metastases in a mixed cohort of patients with initial diagnosis, recurrent disease, and advanced mCRPC. Patient-based sensitivities and specificities were $98.7 \%-100 \%$ and $88.2 \%-100 \%$, respectively, for PSMA PET and $86.7 \%-89.3 \%$ and $60.8 \%-96.1 \%$, respectively, for BS. In the subgroup analysis, PSMA PET outperformed BS specifically in the primary staging cohort (sensitivity, $100 \%$ vs. $57.1 \%$ ).

These findings highlight that although current recommendations favor CT, MRI, and BS for staging of metastatic disease based on robust data on treatment response and prognostic value, newer imaging modalities, such as PSMA PET, have shown higher diagnostic value albeit their association with prognosis and outcome are yet to be determined.

\section{Imaging in BCR and Recurrent Disease After Treatment}

With the increasing use of posttreatment monitoring of PSA (e.g., after radical prostatectomy and radiation therapy), disease recurrence and metastatic state are often being detected earlier, at a time of lower disease burden. Disease recurrence is generally suspected when there is increased PSA levels from previously undetectable (after radical prostatectomy) or nadir (after radiation therapy) levels. Disease recurrence after definitive initial therapy is not rare: between $27 \%$ and $53 \%$ of all patients undergoing 
radical prostatectomy or radiation therapy develop PSA recurrence within 10 years $(58)$.

BCR is not synonymous with the presence of clinical disease and often predates clinically overt disease for months to years. Nevertheless, despite the variability in the natural history of BCR, it may be a window of opportunity for early initiation of salvage therapy. Evidence suggests that in recurrent disease, local therapies such as salvage external-beam radiation therapy after radical prostatectomy are most effective during the early phase of PSA recurrence (59). This suggestion translates into great interest in early imaging-based detection of actionable disease. Imaging plays a pivotal role in the assessment of disease recurrence by providing information on disease presence, volume, and distribution, with profound implications for management and clinical decision making. Although local recurrence can be treated with a combination of salvage radiation therapy and ADT, metastatic disease requires the addition of systemic or targeted therapy of affected regions; the distinction is mainly made based on imaging.

mpMRI is the most widely studied imaging technique for the detection of local recurrence after definitive therapy. The most common site of local recurrence after radical prostatectomy is the neovesicourethral anastomosis (Supplemental Fig. 6) (60). On mpMRI, local recurrence usually presents as a nodular to ill-defined soft-tissue mass of intermediate T2-weighted signal intensity with associated diffusion restriction and rapid, early enhancement on dynamic contrast-enhanced imaging. The distorted anatomy and posttreatment edematous changes after radiation therapy may limit evaluation. In the setting of radiation therapy, diffusionweighted imaging and dynamic contrast-enhanced imaging have been shown to accurately identify local recurrence in the irradiated prostate (Supplemental Fig. 3) (61,62).

In a metaanalysis of the performance of mpMRI for the detection of local recurrence after radical prostatectomy, the pooled sensitivities and specificities were $82 \%$ and $87 \%$, respectively. The pooled sensitivities and specificities after radiation therapy were $82 \%$ and $74 \%$, respectively (63). These findings were supported in another metaanalysis comprising 718 patients (64), with pooled sensitivities and specificities of $84 \%$ and $85 \%$, respectively, for the use of mpMRI to detect local recurrence after radical prostatectomy.

Barchetti et al. (65) evaluated the performance of unenhanced WB MRI for the detection of lesions in the setting of BCR in 152 patients. WB MRI detected LN metastases with a sensitivity, specificity, positive predictive value, negative predictive value, and accuracy of $98 \%, 99 \%, 97 \%, 98 \%$, and $98 \%$, respectively, and for osseous metastases the respective results were $99 \%, 98 \%, 98 \%$, $96 \%$, and $98 \%$. Although the inclusion criterion of a post-radical prostatectomy PSA level of at least $1.2 \mathrm{ng} / \mathrm{mL}$ was stated, the mean PSA values of the included patients were not reported.

There are distinct clinical scenarios of local recurrence in which PSMA PET/MRI could increase the detectability of MR-occult lesions, such as after brachytherapy, when the artifacts associated with the seeds may degrade image quality and limit evaluation. Another use for PSMA PET/MRI could be the assessment of local recurrence after focal ablation therapy (e.g., high-intensity focused ultrasound or cryoablation) (Fig. 3) (66).

A limitation of the currently most widely used PET tracer, ${ }^{68} \mathrm{Ga}$-PSMA-11, is its urinary excretion and high activity within the urinary bladder (67). The development of novel tracers, such as the recently introduced ${ }^{18} \mathrm{~F}$-PSMA-1007, which is predominantly excreted via the hepatobiliary route, could thus improve

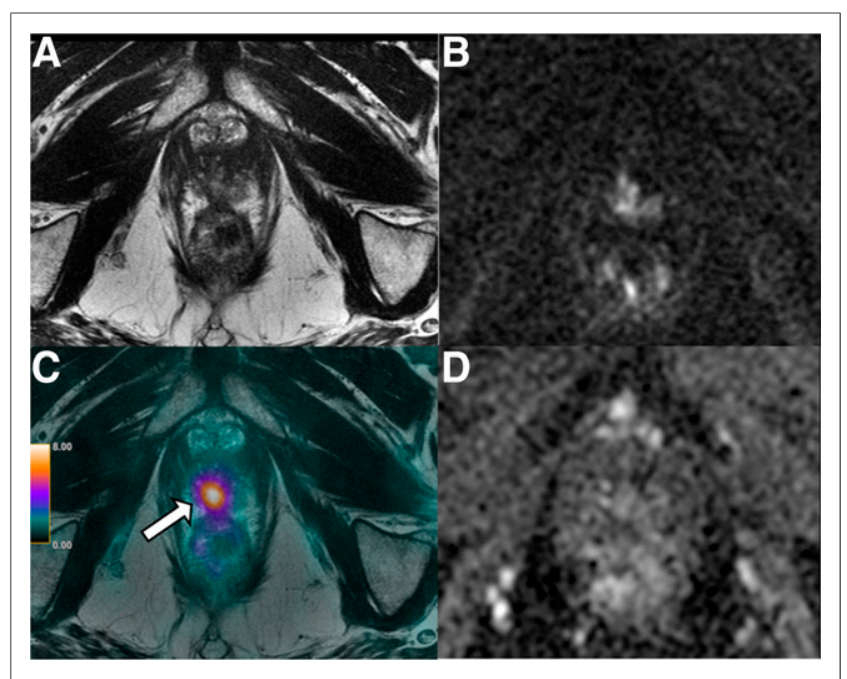

FIGURE 3. A 69-y-old patient with history of Gleason 7 PCa treated with high-intensity focus ultrasound therapy 1 year previously. (A, B, and D) Surveillance mpMRI does not reveal any findings concerning for recurrence. (C) On ${ }^{68} \mathrm{Ga}-\mathrm{PSMA} \mathrm{PET} / \mathrm{MRI}$, area of focal intense uptake localized to right apex (arrow) is seen, corresponding to subsequently biopsy-proven recurrent Gleason $4+4$ tumor.

the evaluation for locoregional recurrence and small pelvic LNs near the ureter.

Although ${ }^{18} \mathrm{~F}$-fluoromethylcholine, ${ }^{11} \mathrm{C}$-choline, and ${ }^{18} \mathrm{~F}$-fluciclovine PET/CT are used in recurrent $\mathrm{PCa}$, they have limited performance at low PSA levels - the window of opportunity when salvage treatment strategies are most efficient. Recent data suggest that imaging with PSMA-targeting radioligands is sensitive for the detection of lesions in BCR, even at low PSA levels (Fig. 4) (68).

In a recent metaanalysis including 43 studies with 5,113 patients, Tan et al. (69) analyzed the performance of PSMA-targeted radiotracers for the detection of BCR stratified by PSA level. The pooled detection rate was $70.2 \%$ for the entire cohort, ranging from $44.9 \%$ for a PSA level of less than $0.5 \mathrm{ng} / \mathrm{mL}$ to $93.9 \%$ for a PSA level of at least $2 \mathrm{ng} / \mathrm{mL}$ on stratified subgroup analysis. All the studies showed a maximal detection rate at a PSA level of at

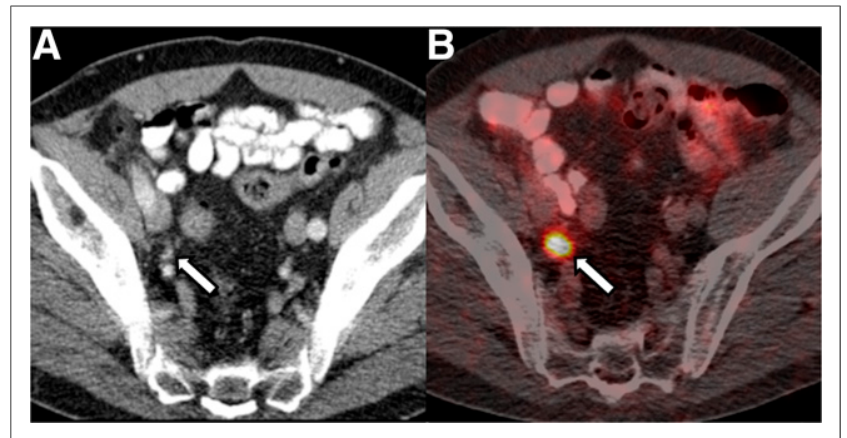

FIGURE 4. A 68-y-old patient with BCR of Gleason $3+4 \mathrm{PCa}$ after radical prostatectomy and pelvic $L N$ dissection 11 years previously. PSA was $1.4 \mathrm{ng} / \mathrm{mL}$ at time of imaging. (A) CT portion of study does not reveal any abnormal LNs. (B) Fused ${ }^{68} \mathrm{Ga}-\mathrm{PSMA}$ PET/CT shows focal avid uptake in right external iliac region (arrow) corresponding to subcentimeter external iliac LN (arrow in A), suggestive of LN metastasis, which was confirmed on subsequent excisional biopsy. 
least $2 \mathrm{ng} / \mathrm{mL}$, with rates of around $90 \%$, as is consistent with the fact that around $5 \%-10 \%$ of PCa do not overexpress PSMA and are thus PSMA-negative (70). The authors concluded that PSMAtargeted radiotracers are likely effective for the detection of BCR at low PSA levels, but there was significant study heterogeneity ( $\mathrm{I}^{2}$ of $95.6 \%$ for the entire cohort) due to differences in inclusion criteria, study populations, methodology, radiotracers used, retrospective designs, and reference standards.

In the LOCATE trial (71), a prospective, single-arm multicenter trial including 213 men with BCR (median PSA level, $1.0 \mathrm{ng} / \mathrm{mL}$; range, $0.2-93.5 \mathrm{ng} / \mathrm{mL}$ ), the detection rate and impact of ${ }^{18} \mathrm{~F}-\mathrm{flu}-$ ciclovine PET/CT on clinical management were evaluated. In 59\% of patients, clinical management was changed when the results of the ${ }^{18} \mathrm{~F}$-fluciclovine PET/CT were obtained; of those cases, $78 \%$ were classified as a major change-namely, change in treatment modality. Detection rates were compared with that of conventional imaging (BS and CT or MRI). The overall per-patient detection rate for ${ }^{18} \mathrm{~F}$-fluciclovine was $57 \%$, with a positive correlation with PSA level $(50 \%$ at a level of $0.5-1.0 \mathrm{ng} / \mathrm{mL}$, dropping to $31 \%$ at a level of $<0.5 \mathrm{ng} / \mathrm{mL}$ ). The improved detection rate of ${ }^{18} \mathrm{~F}$-fluciclovine PET/CT, when compared with conventional imaging modalities, is not surprising given the well-known limitations of the latter in the setting of BCR.

In a retrospective trial by Jilg et al. (72), the detection rate of LN metastases by ${ }^{18} \mathrm{~F}$-choline PET/CT was compared with that by ${ }^{68} \mathrm{Ga}$-PSMA using histopathology from salvage pelvic lymph node dissection as a reference standard. The investigators specifically examined the PET detection rate in relation to the size of LN tumor deposits. ${ }^{68} \mathrm{Ga}$-PSMA PET/CT was superior to ${ }^{18} \mathrm{~F}$-choline $\mathrm{PET} / \mathrm{CT}$ in the detection of smaller LN metastases, with a detection rate of $90 \%$ at 7.4 and $4.9 \mathrm{~mm}$ (long and short diameters) for ${ }^{68} \mathrm{Ga}$-PSMA PET/CT, compared with of $90 \%$ at 11.2 and $6.3 \mathrm{~mm}$ for ${ }^{18} \mathrm{~F}$ choline PET/CT, highlighting the higher sensitivity of PSMA PET/CT for small LN metastases

An important prospective study substantiating the high performance of PSMA PET in the setting of recurrent disease was published recently by Fendler et al. (73). This study included 635 men and used a composite reference standard and an independent masked multireader analysis. Their results showed not only high detection rates and positive predictive value $(84 \%$ or $92 \%$, depending on reference standard) but also high interreader agreement for localization of recurrent PCa even at low PSA levels (detection rate of $84 \%$ for PSA level $<2 \mathrm{ng} / \mathrm{mL}$ ).

Although PSMA-targeting radiotracers are appealing given their diagnostic performance and potential for combined diagnostic and therapeutic use, there are no published formal allencompassing prospective trials comparing the performance of PSMA tracers with other tracers head to head. It is expected that the evidence for PSMA will be strengthened by results from ongoing prospective trials, such as the phase II prospective trials comparing ${ }^{68} \mathrm{Ga}-\mathrm{PSMA}-11 \mathrm{PET} / \mathrm{CT}$ with ${ }^{18} \mathrm{~F}$-fluciclovine PET/CT in the setting of primary PCa and BCR (NCT03762759 and NCT03515577). Preliminary results from one of these studies by Calais et al. (74) showed prospective detection rates for PSMA to be double those for ${ }^{18} \mathrm{~F}$-fluciclovine in patients with BCR after radical prostatectomy at low PSA levels $(\leq 2.0 \mathrm{ng} / \mathrm{mL})$.

\section{Imaging MCRPC}

mCRPC is an advanced form of PCa defined by the development of resistance to conventional ADT. Most patients with mCRPC develop bone metastases. Further, the fact that visceral metastases are more common in MCRPC and after multiple lines of therapy is thought to correlate with emergent aggressive histologic variants.

AR-targeted imaging with ${ }^{18} \mathrm{~F}$-FDHT is an interesting area of current research for pretreatment stratification and therapeutic response assessment in castration-resistant patients evaluated for newer, high-affinity AR inhibitor therapy (e.g., enzalutamide) (75). In combination with ${ }^{18} \mathrm{~F}-\mathrm{FDG},{ }^{18} \mathrm{~F}$-FDHT PET/CT may also be a useful marker of PCa heterogeneity. In a study by Fox et al. (76) including 133 patients with mCRPC undergoing dual ${ }^{18} \mathrm{~F}$ FDG and ${ }^{18}$ F-FDHT PET/CT, metastatic lesions were grouped into 4 different phenotypes based on the evaluation of metabolic activity with the 2 different tracers. Patients with predominantly ${ }^{18}$ F-FDHT-negative and ${ }^{18}$ F-FDG-positive lesions had the poorest prognosis, leading to the assumption that those patients had biologically more aggressive tumors without significant expression of wild-type AR (which would be actionable with antiandrogenic therapy) (76). This result suggests that the presence of ${ }^{18} \mathrm{~F}-\mathrm{FDG}-$ avid disease is a surrogate marker of poor prognosis and aggressive biology.

\section{FUTURE OUTLOOK: THERANOSTICS}

Beyond diagnostic capabilities, PSMA-based imaging has direct therapeutic implications. Targeted radioligand therapy (RLT) with PSMA is an attractive, quickly evolving therapeutic option for advanced mCRPC, as PSMA expression is particularly high in castration-resistant metastases after multiple lines of therapy (77). The rationale is to "see what you treat" by combining a diagnostic with a therapeutic radioligand. ${ }^{177} \mathrm{Lu}-\mathrm{PSMA}-617$ and ${ }^{177} \mathrm{Lu}-$ PSMA I\&T are small-molecule inhibitors of PSMA that can be used therapeutically. ${ }^{177} \mathrm{Lu}$ decays through $\beta$-emission and lowenergy $\gamma$-photons; the former is used therapeutically whereas the latter is used for imaging. RLT with ${ }^{177} \mathrm{Lu}$-PSMA is not yet approved by the Food and Drug Administration or by the European Medicines Agency but has been shown to have favorable safety and toxicity profiles (78). Further, data from prospective phase 2 trials have shown high response rates in patients with mCRPC treated with PSMA RLT who had progressed after conventional treatments (79). The efficacy of PSMA RLT is currently being evaluated in an ongoing randomized phase 3 trial (VISION) for subsequent approval evaluation.

Nonetheless, results evaluating the theranostics approach in $\mathrm{PCa}$ appear promising. In a systematic review, von Eyben et al. (80) compared ${ }^{177} \mathrm{Lu}-\mathrm{RLT}$ with other third-line treatments in mCRPC, including 12 studies with 669 patients and 16 studies with 1,338 patients. ${ }^{177} \mathrm{Lu}$-PSMA RLT more frequently yielded a PSA decline of at least $50 \%$ than did third-line treatment (mean, $44 \%$ vs. $22 \%$; $P=0.0002$ ). Further, the adverse event-related discontinuation rate was higher for third-line treatments than for ${ }^{177} \mathrm{Lu}-\mathrm{PSMA}$ RLT $(P<0.001)$. Results from several international ongoing prospective trials should help elucidate the value of ${ }^{177} \mathrm{Lu}$-PSMA RLT in the management of mCRPC (phase III VISION trial [NCT03511664, NCT03454750], phase II TheraP trial [NCT03392428]). The VISION trial will evaluate the outcomes of patients with mCRPC treated with ${ }^{177}$ Lu-PSMA-617 compared with the best standard care.

\section{ACKNOWLEDGMENTS}

We thank Joanne Chin and Garon Scott for their editorial support on the manuscript. 


\section{REFERENCES}

1. Yadav SS, Stockert JA, Hackert V, Yadav KK, Tewari AK. Intratumor heterogeneity in prostate cancer. Urol Oncol. 2018;36:349-360.

2. Pezaro C, Omlin A, Lorente D, et al. Visceral disease in castration-resistant prostate cancer. Eur Urol. 2014;65:270-273.

3. Turkbey B, Rosenkrantz AB, Haider MA, et al. Prostate imaging reporting and data system version 2.1: 2019 update of prostate imaging reporting and data system version 2. European Urology.

4. Turkbey B, Mani H, Shah V, et al. Multiparametric 3T prostate magnetic resonance imaging to detect cancer: histopathological correlation using prostatectomy specimens processed in customized magnetic resonance imaging based molds. J Urol. 2011;186:1818-1824.

5. Weinreb JC, Barentsz JO, Choyke PL, et al. PI-RADS prostate imaging: reporting and data system-2015, version 2. Eur Urol. 2016;69:16-40.

6. Fanti S, Minozzi S, Antoch G, et al. Consensus on molecular imaging and theranostics in prostate cancer. Lancet Oncol. 2018;19:e696-e708.

7. Padhani AR, Lecouvet FE, Tunariu N, et al. METastasis Reporting and Data System for Prostate Cancer: practical guidelines for acquisition, interpretation, and reporting of whole-body magnetic resonance imaging-based evaluations of multiorgan involvement in advanced prostate cancer. Eur Urol. 2017;71:81-92.

8. Metser U, Chua SS, Ho B, et al. The contribution of multiparametric pelvic \& whole body MR to interpretation of ${ }^{18} \mathrm{~F}$-fluoromethylcholine or ${ }^{68} \mathrm{Ga}-\mathrm{HBED}-\mathrm{CC}$ PSMA-11 PET/CT in patients with biochemical failure following radical prostatectomy. J Nucl Med. March 22, 2019 [Epub ahead of print].

9. Zacho HD, Nielsen JB, Afshar-Oromieh A, et al. Prospective comparison of ${ }^{68} \mathrm{Ga}$ PSMA PET/CT, ${ }^{18} \mathrm{~F}$-sodium fluoride PET/CT and diffusion weighted-MRI at for the detection of bone metastases in biochemically recurrent prostate cancer. Eur J Nucl Med Mol Imaging. 2018;45:1884-1897.

10. Padhani AR, Lecouvet FE, Tunariu N, et al. Rationale for modernising imaging in advanced prostate cancer. Eur Urol Focus. 2017;3:223-239.

11. Donohoe KJ, Cohen EJ, Giammarile F, et al. Appropriate use criteria for bone scintigraphy in prostate and breast cancer: summary and excerpts. J Nucl Med. 2017;58(4):14N-17N.

12. Wibmer AG, Burger IA, Sala E, Hricak H, Weber WA, Vargas HA. Molecular imaging of prostate cancer. Radiographics. 2016;36:142-159.

13. Jadvar H. Is there use for FDG-PET in prostate cancer? Semin Nucl Med. 2016;46: 502-506.

14. Vargas HA, Wassberg C, Fox JJ, et al. Bone metastases in castration-resistant prostate cancer: associations between morphologic CT patterns, glycolytic activity, and androgen receptor expression on PET and overall survival. Radiology. 2014;271:220-229.

15. Shen G, Liu J, Hao Z, Huang R, Kuang A. Is ${ }^{18}$ F-FDG PET/CT a useful tool in prostate cancer? [abstract]. J Nucl Med. 2017;58(suppl 1):1077.

16. Segall G, Delbeke D, Stabin MG, et al. SNM practice guideline for sodium ${ }^{18} \mathrm{~F}$ fluoride PET/CT bone scans 1.0. J Nucl Med. 2010;51:1813-1820.

17. Löfgren J, Mortensen J, Rasmussen SH, et al. A prospective study comparing ${ }^{99 \mathrm{~m}} \mathrm{Tc}$-hydroxyethylene-diphosphonate planar bone scintigraphy and whole-body SPECT/CT with ${ }^{18}$ F-fluoride PET/CT and ${ }^{18}$ F-fluoride PET/MRI for diagnosing bone metastases. J Nucl Med. 2017;58:1778-1785.

18. Schwarzenböck S, Souvatzoglou M, Krause BJ. Choline PET and PET/CT in primary diagnosis and staging of prostate cancer. Theranostics. 2012;2:318-330.

19. Hodolic M. Role of ${ }^{18} \mathrm{~F}$-choline PET/CT in evaluation of patients with prostate carcinoma. Radiol Oncol. 2011;45:17-21.

20. Schuster DM, Savir-Baruch B, Nieh PT, et al. Detection of recurrent prostate carcinoma with anti-1-amino-3- ${ }^{18}$ F-fluorocyclobutane-1-carboxylic acid PET/CT and ${ }^{111}$ In-capromab pendetide SPECT/CT. Radiology. 2011;259:852-861.

21. Bostwick DG, Pacelli A, Blute M, Roche P, Murphy GP. Prostate specific membrane antigen expression in prostatic intraepithelial neoplasia and adenocarcinoma: a study of 184 cases. Cancer. 1998;82:2256-2261.

22. Oh SW, Cheon GJ. Prostate-specific membrane antigen PET imaging in prostate cancer: opportunities and challenges. Korean J Radiol. 2018;19:819-831.

23. Kesler M, Levine C, Hershkovitz D, et al. ${ }^{68} \mathrm{Ga}$-PSMA is a novel PET-CT tracer for imaging of hepatocellular carcinoma: a prospective pilot study. J Nucl Med. July 12, 2018 [Epub ahead of print].

24. Heitkötter B, Steinestel K, Trautmann M, et al. Neovascular PSMA expression is a common feature in malignant neoplasms of the thyroid. Oncotarget. 2018;9: 9867-9874.

25. Chang SS, O'Keefe DS, Bacich DJ, Reuter VE, Heston WD, Gaudin PB. Prostate-specific membrane antigen is produced in tumor-associated neovasculature. Clin Cancer Res. 1999;5:2674-2681.

26. Giesel FL, Knorr K, Spohn F, et al. Detection efficacy of ${ }^{18}$ F-PSMA-1007 PET/ $\mathrm{CT}$ in 251 patients with biochemical recurrence of prostate cancer after radical prostatectomy. J Nucl Med. 2019;60:362-368.
27. Rathkopf D, Scher HI. Androgen receptor antagonists in castration-resistant prostate cancer. Cancer J. 2013;19:43-49.

28. Ceder Y, Bjartell A, Culig Z, Rubin MA, Tomlins S, Visakorpi T. The molecular evolution of castration-resistant prostate cancer. Eur Urol Focus. 2016;2:506-513.

29. Larson SM, Morris M, Gunther I, et al. Tumor localization of $16 \beta^{-18} \mathrm{~F}$-fluoro- $5 \alpha-$ dihydrotestosterone versus ${ }^{18} \mathrm{~F}-\mathrm{FDG}$ in patients with progressive, metastatic prostate cancer. J Nucl Med. 2004;45:366-373.

30. Vargas HA, Kramer GM, Scott AM, et al. Reproducibility and repeatability of semiquantitative ${ }^{18} \mathrm{~F}$-fluorodihydrotestosterone uptake metrics in castration-resistant prostate cancer metastases: a prospective multicenter study. J Nucl Med. 2018;59: 1516-1523.

31. Eiber M, Weirich G, Holzapfel K, et al. Simultaneous ${ }^{68} \mathrm{Ga}$-PSMA HBED-CC PET/MRI improves the localization of primary prostate cancer. Eur Urol. 2016;70: 829-836.

32. Park SY, Zacharias C, Harrison C, et al. Gallium 68 PSMA-11 PET/MR imaging in patients with intermediate- or high-risk prostate cancer. Radiology. 2018;288:495-505.

33. Hicks RM, Simko JP, Westphalen AC, et al. Diagnostic accuracy of ${ }^{68}$ Ga-PSMA11 PET/MRI compared with multiparametric MRI in the detection of prostate cancer. Radiology. 2018;289:730-737.

34. Ahmed HU, El-Shater Bosaily A, Brown LC, et al. Diagnostic accuracy of multiparametric MRI and TRUS biopsy in prostate cancer (PROMIS): a paired validating confirmatory study. Lancet. 2017;389:815-822.

35. Kasivisvanathan V, Rannikko AS, Borghi M, et al. MRI-targeted or standard biopsy for prostate-cancer diagnosis. N Engl J Med. 2018;378:1767-1777.

36. Moldovan PC, Van den Broeck T, Sylvester R, et al. What is the negative predictive value of multiparametric magnetic resonance imaging in excluding prostate cancer at biopsy? A systematic review and meta-analysis from the European Association of Urology Prostate Cancer Guidelines Panel. Eur Urol. 2017;72:250-266.

37. Mohammadian Bajgiran A, Afshari Mirak S, Shakeri S, et al. Characteristics of missed prostate cancer lesions on 3T multiparametric-MRI in 518 patients: based on PI-RADSv2 and using whole-mount histopathology reference. Abdom Radiol (NY). 2019;44:1052-1061.

38. Borofsky S, George AK, Gaur S, et al. What are we missing? False-negative cancers at multiparametric MR imaging of the prostate. Radiology. 2018;286:186-195.

39. Sala E, Akin O, Moskowitz CS, et al. Endorectal MR imaging in the evaluation of seminal vesicle invasion: diagnostic accuracy and multivariate feature analysis. Radiology. 2006;238:929-937.

40. de Rooij M, Hamoen EH, Witjes JA, Barentsz JO, Rovers MM. Accuracy of magnetic resonance imaging for local staging of prostate cancer: a diagnostic metaanalysis. Eur Urol. 2016;70:233-245.

41. Mühlematter UJ, Becker KSA, Müller J, et al. Detection of extracapsular extension in high risk prostate cancer patients: multiparametric MRI vs. ${ }^{68} \mathrm{Ga}$-PSMA11 PET/MRI. Paper presented at: 2019 European Congress of Radiology; February 28, 2019; Vienna, Austria.

42. Ross HM, Kryvenko ON, Cowan JE, Simko JP, Wheeler TM, Epstein JI. Do adenocarcinomas of the prostate with Gleason score (GS) $\leq 6$ have the potential to metastasize to lymph nodes? Am J Surg Pathol. 2012;36:1346-1352.

43. Eggener SE, Scardino PT, Walsh PC, et al. Predicting 15-year prostate cancer specific mortality after radical prostatectomy. J Urol. 2011;185:869-875.

44. Briganti A, Larcher A, Abdollah F, et al. Updated nomogram predicting lymph node invasion in patients with prostate cancer undergoing extended pelvic lymph node dissection: the essential importance of percentage of positive cores. Eur Urol. 2012;61:480-487.

45. Torabi M, Aquino SL, Harisinghani MG. Current concepts in lymph node imaging. J Nucl Med. 2004;45:1509-1518.

46. Hövels AM, Heesakkers RAM, Adang EM, et al. The diagnostic accuracy of CT and MRI in the staging of pelvic lymph nodes in patients with prostate cancer: a meta-analysis. Clin Radiol. 2008;63:387-395.

47. Woo S, Suh CH, Kim SY, Cho JY, Kim SH. The diagnostic performance of MRI for detection of lymph node metastasis in bladder and prostate cancer: an updated systematic review and diagnostic meta-analysis. AJR. 2018;210:W95-W109.

48. Evangelista L, Guttilla A, Zattoni F, Muzzio PC, Zattoni F. Utility of choline positron emission tomography/computed tomography for lymph node involvement identification in intermediate- to high-risk prostate cancer: a systematic literature review and meta-analysis. Eur Urol. 2013;63:1040-1048.

49. Selnæs KM, Kruger-Stokke B, Elschot M, et al. ${ }^{18} \mathrm{~F}$-fluciclovine PET/MRI for preoperative lymph node staging in high-risk prostate cancer patients. Eur Radiol. 2018;28:3151-3159.

50. Hofman MS, Murphy DG, Williams SG, et al. A prospective randomized multicentre study of the impact of gallium-68 prostate-specific membrane antigen (PSMA) PET/CT imaging for staging high-risk prostate cancer prior to curative-intent surgery or radiotherapy (proPSMA study): clinical trial protocol. $B J U$ Int. 2018;122:783-793. 
51. Grubmüller B, Baltzer P, Hartenbach S, et al. PSMA ligand PET/MRI for primary prostate cancer: staging performance and clinical impact. Clin Cancer Res. 2018;24:6300-6307.

52. Maurer T, Gschwend JE, Rauscher I, et al. Diagnostic efficacy of ${ }^{68}$ gallium-PSMA positron emission tomography compared to conventional imaging for lymph node staging of 130 consecutive patients with intermediate to high risk prostate cancer. J Urol. 2016;195:1436-1443.

53. Giesel FL, Fiedler H, Stefanova M, et al. PSMA PET/CT with Glu-urea-Lys(Ahx)- $\left[{ }^{68} \mathrm{Ga}(\mathrm{HBED}-\mathrm{CC})\right]$ versus 3D CT volumetric lymph node assessment in recurrent prostate cancer. Eur J Nucl Med Mol Imaging. 2015;42:1794-1800.

54. Armstrong AJ, Anand A, Edenbrandt L, et al. Phase 3 assessment of the automated bone scan index as a prognostic imaging biomarker of overall survival in men with metastatic castration-resistant prostate cancer: a secondary analysis of a randomized clinical trial. JAMA Oncol. 2018;4:944-951.

55. Scher HI, Morris MJ, Stadler WM, et al. Trial design and objectives for castration-resistant prostate cancer: updated recommendations from the Prostate Cancer Clinical Trials Working Group 3. J Clin Oncol. 2016;34:1402-1418.

56. Sweeney CJ, Chen YH, Carducci M, et al. Chemohormonal therapy in metastatic hormone-sensitive prostate cancer. N Engl J Med. 2015;373:737-746.

57. Pyka T, Okamoto S, Dahlbender M, et al. Comparison of bone scintigraphy and ${ }^{68} \mathrm{Ga}$-PSMA PET for skeletal staging in prostate cancer. Eur J Nucl Med Mol Imaging. 2016;43:2114-2121.

58. Mottet N, Bellmunt J, Bolla M, et al. EAU-ESTRO-SIOG guidelines on prostate cancer. Part 1: screening, diagnosis, and local treatment with curative intent. Eur Urol. 2017;71:618-629.

59. Briganti A, Karnes RJ, Joniau S, et al. Prediction of outcome following early salvage radiotherapy among patients with biochemical recurrence after radical prostatectomy. Eur Urol. 2014;66:479-486.

60. Oppenheimer DC, Weinberg EP, Hollenberg GM, Meyers SP. Multiparametric magnetic resonance imaging of recurrent prostate cancer. J Clin Imaging Sci. 2016;6:18.

61. Donati OF, Jung SI, Vargas HA, et al. Multiparametric prostate MR imaging with T2-weighted, diffusion-weighted, and dynamic contrast-enhanced sequences: are all pulse sequences necessary to detect locally recurrent prostate cancer after radiation therapy? Radiology. 2013;268:440-450.

62. Akin O, Gultekin DH, Vargas HA, et al. Incremental value of diffusion weighted and dynamic contrast enhanced MRI in the detection of locally recurrent prostate cancer after radiation treatment: preliminary results. Eur Radiol. 2011;21:19701978.

63. Wu LM, Xu JR, Gu HY, et al. Role of magnetic resonance imaging in the detection of local prostate cancer recurrence after external beam radiotherapy and radical prostatectomy. Clin Oncol (R Coll Radiol). 2013;25:252-264.

64. Sandgren K, Westerlinck P, Jonsson JH, et al. Imaging for the detection of locoregional recurrences in biochemical progression after radical prostatectomy: a systematic review. Eur Urol Focus. November 11, 2017 [Epub ahead of print].

65. Barchetti F, Stagnitti A, Megna V, et al. Unenhanced whole-body MRI versus PET-CT for the detection of prostate cancer metastases after primary treatment. Eur Rev Med Pharmacol Sci. 2016;20:3770-3776.
66. Burger IA, Müller J, Donati OF, et al. ${ }^{68} \mathrm{Ga}$-PSMA-11 PET/MR detects local recurrence occult on mpMRI in prostate cancer patients after HIFU. J Nucl Med. 2019;60:1118-1123.

67. Freitag MT, Radtke JP, Afshar-Oromieh A, et al. Local recurrence of prostate cancer after radical prostatectomy is at risk to be missed in ${ }^{68} \mathrm{Ga}-\mathrm{PSMA}-11$-PET of PET/CT and PET/MRI: comparison with mpMRI integrated in simultaneous PET/MRI. Eur J Nucl Med Mol Imaging. 2017;44:776-787.

68. Afshar-Oromieh A, Zechmann CM, Malcher A, et al. Comparison of PET imaging with a ${ }^{68} \mathrm{Ga}$-labelled PSMA ligand and ${ }^{18} \mathrm{~F}$-choline-based PET/CT for the diagnosis of recurrent prostate cancer. Eur J Nucl Med Mol Imaging. 2014;41:11-20.

69. Tan N, Bavadian N, Calais J, et al. Imaging of prostate specific membrane antigen targeted radiotracers for the detection of prostate cancer biochemical recurrence after definitive therapy: a systematic review and meta-analysis. $J$ Urol. 2019;202:231-240.

70. Bravaccini S, Puccetti M, Bocchini M, et al. PSMA expression: a potential ally for the pathologist in prostate cancer diagnosis. Sci Rep. 2018;8:4254.

71. Andriole GL, Kostakoglu L, Chau A, et al. The impact of positron emission tomography with ${ }^{18} \mathrm{~F}$-fluciclovine on the treatment of biochemical recurrence of prostate cancer: results from the LOCATE trial. J Urol. 2019;201:322-331.

72. Jilg CA, Drendel V, Rischke HC, et al. Detection rate of ${ }^{18} \mathrm{~F}$-choline PET/CT and ${ }^{68} \mathrm{Ga}$-PSMA-HBED-CC PET/CT for prostate cancer lymph node metastases with direct link from PET to histopathology: dependence on the size of tumor deposits in lymph nodes. J Nucl Med. 2019;60:971-977.

73. Fendler WP, Calais J, Eiber M, et al. Assessment of ${ }^{68}$ Ga-PSMA-11 PET accuracy in localizing recurrent prostate cancer: a prospective single-arm clinical trial. JAMA Oncol. 2019;5:856-863.

74. Calais J, Ceci F, Nguyen K, et al. Prospective head-to-head comparison of ${ }^{18} \mathrm{~F}-$ fluciclovine and ${ }^{68} \mathrm{Ga}$-PSMA-11 PET/CT for localization of prostate cancer biochemical recurrence after primary prostatectomy [abstract]. J Clin Oncol. 2019; 37(suppl): 15 .

75. Pandit-Taskar N, Veach DR, Fox JJ, Scher HI, Morris MJ, Larson SM. Evaluation of castration-resistant prostate cancer with androgen receptor-axis imaging. J Nucl Med. 2016;57(suppl):73S-78S.

76. Fox JJ, Gavane SC, Blanc-Autran E, et al. Positron emission tomography/computed tomography-based assessments of androgen receptor expression and glycolytic activity as a prognostic biomarker for metastatic castration-resistant prostate cancer. JAMA Oncol. 2018;4:217-224.

77. Fendler WP, Rahbar K, Herrmann K, Kratochwil C, Eiber M. ${ }^{177}$ Lu-PSMA radioligand therapy for prostate cancer. J Nucl Med. 2017;58:1196-1200.

78. Rahbar K, Ahmadzadehfar H, Kratochwil C, et al. German multicenter study investigating ${ }^{177} \mathrm{Lu}-\mathrm{PSMA}-617$ radioligand therapy in advanced prostate cancer patients. J Nucl Med. 2017;58:85-90.

79. Hofman MS, Violet J, Hicks RJ, et al. $\left[{ }^{177} \mathrm{Lu}\right]-P S M A-617$ radionuclide treatment in patients with metastatic castration-resistant prostate cancer (LuPSMA trial): a single-centre, single-arm, phase 2 study. Lancet Oncol. 2018;19:825-833.

80. von Eyben FE, Roviello G, Kiljunen T, et al. Third-line treatment and ${ }^{177} \mathrm{Lu}-$ PSMA radioligand therapy of metastatic castration-resistant prostate cancer: a systematic review. Eur J Nucl Med Mol Imaging. 2018;45:496-508. 